Original Article

\title{
Prognostic role of atrial fibrillation in patients affected by chronic heart failure. Data from the MECKI score research group
}

\author{
Stefania Paolillo a , Piergiuseppe Agostoni b,c,*, Daniele Masarone d, Ugo Corrà e , Claudio Passino f,g,
} Domenico Scrutinio $^{\text {h }}$, Michele Correale ${ }^{\mathrm{i}}$, Gaia Cattadori ${ }^{\mathrm{b}}$, Marco Metra ${ }^{\mathrm{j}}$, Davide Girola ${ }^{\mathrm{k}}$, Massimo F. Piepoli ${ }^{1}$, Elisabetta Salvioni ${ }^{\mathrm{b}}$, Marta Giovannardi ${ }^{\mathrm{b}}$, Annamaria Iorio ${ }^{\mathrm{m}}$, Michele Emdin ${ }^{\mathrm{f}}$, Rosa Raimondo ${ }^{\mathrm{n}}$, Federica Re $^{\mathrm{o}}$, Mariantonietta Cicoira ${ }^{\mathrm{p}}$, Romualdo Belardinelli ${ }^{\mathrm{q}}$, Marco Guazzi ${ }^{\mathrm{r}}$, Francesco Clemenza ${ }^{\mathrm{s}}$, Gianfranco Parati ${ }^{\mathrm{t}}$, Angela B. Scardovi ${ }^{\text {u }}$, Andrea Di Lenarda v, Rocco La Gioia ${ }^{\mathrm{h}}$, Maria Frigerio ${ }^{\mathrm{k}}$, Carlo Lombardi ${ }^{\mathrm{i}}$, Paola Gargiulo ${ }^{\mathrm{w}}$, Gianfranco Sinagra ${ }^{m}$, Giuseppe Pacileo ${ }^{\mathrm{d}}$, Pasquale Perrone-Filardi ${ }^{\mathrm{a}}$, Giuseppe Limongelli ${ }^{\mathrm{d}}$, On behalf of the Metabolic Exercise test data combined with Cardiac and Kidney Indexes (MECKI) Score Research Group (see Appendix A)

a Department of Advanced Biomedical Sciences, “Federico II" University, Napoli, Italy

b Centro Cardiologico Monzino, IRCCS, Milano, Italy

c Department of Clinical Sciences and Community Health, Università di Milano, Milano, Italy

d Cardiologia SUN, Ospedale Monaldi (Azienda dei Colli), Seconda Università di Napoli, Napoli, Italy

e Divisione di Cardiologia Riabilitativa, Fondazione Salvatore Maugeri, IRCCS, Istituto Scientifico di Veruno, Veruno, Italy

${ }^{\mathrm{f}}$ Fondazione Gabriele Monasterio, CNR-Regione Toscana, Pisa, Italy

' Scuola Superiore S. Anna, Pisa, Italy

h Division of Cardiology, "S. Maugeri” Foundation, IRCCS, Institute of Cassano Murge, Bari, Italy

i Department of Cardiology, University of Foggia, Foggia, Italy

j Cardiology, Department of Medical and Surgical Specialties, Radiological Sciences and Public Health, University of Brescia, Brescia, Italy

k Dipartimento Cardiologico “A. De Gasperis", Ospedale Cà Granda-A.O. Niguarda, Milano, Italy

${ }^{1}$ UOC Cardiologia, G da Saliceto Hospital, Piacenza, Italy

${ }^{m}$ Cardiovascular Department, Ospedali Riuniti and University of Trieste, Trieste, Italy

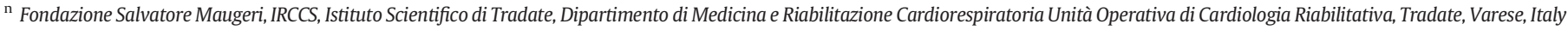

o Cardiology Division, Cardiac Arrhythmia Center and Cardiomyopathies Unit, San Camillo-Forlanini Hospital, Roma, Italy

p Section of Cardiology, Department of Medicine, University of Verona, Italy

q Cardiologia Riabilitativa, Azienda Ospedali Riuniti, Ancona, Italy

${ }^{\mathrm{r}}$ Heart Failure Unit, IRCCS Policlinico San Donato, San Donato Milanese, Italy

${ }^{s}$ Heart Failure Unit, ISMETT (Mediterranean Institute for Transplantation and Advanced Specialized Therapies), Palermo, Italy

${ }^{\mathrm{t}}$ Department of Health Science, University of Milano Bicocca E' Department of Cardiology, San Luca Hospital, Istituto Auxologico Italiano, Milano, Italy

u Cardiology Division, Santo Spirito Hospital, Roma, Italy

${ }^{v}$ Centro Cardiovascolare, Azienda per i Servizi Sanitari no. 1, Trieste, Italy

w SDN Foundation, Institute of Diagnostic and Nuclear Development, Napoli, Italy

\section{A R T I C L E I N F O}

\section{Article history:}

Received 14 February 2015

Received in revised form 22 April 2015

Accepted 27 April 2015

Available online 27 May 2015

\section{Keywords:}

Atrial fibrillation

Heart failure

Prognosis

Matching analysis

MECKI score

\begin{abstract}
A B S T R A C T
Background: Atrial fibrillation (AF) is common in heart failure (HF). It is unclear whether AF has an independent prognostic role in HF. The aim of the present study was to assess the prognostic role of AF in HF patients with reduced ejection fraction $(\mathrm{EF})$.

Methods: HF patients were followed in 17 centers for 3.15 years (1.51-5.24). Study endpoints were the composite of cardiovascular (CV) death and heart transplant (HTX) and all-cause death. Data analysis was performed considering the entire population and a 1 to 1 match between sinus rhythm (SR) and AF patients. Match process was done for age \pm 5 , gender, left ventricle $\mathrm{EF} \pm 5$, peakVO $\mathrm{V}_{2} \pm 3(\mathrm{ml} / \mathrm{min} / \mathrm{kg})$ and recruiting center.

Results: A total of 3447 patients $(S R=2882, A F=565)$ were included in the study. Considering the entire population, CV death and HTX occurred in 114 (20\%) AF vs. 471 (16\%) SR ( p = 0.026) and all-cause death in 130 (23\%) AF vs. $554(19.2 \%)$ SR patients $(\mathrm{p}=0.039)$. At univariable Cox analysis, AF was significantly related to prognosis. Applying a multivariable model based on all variables significant at univariable analysis $\left(\mathrm{EF}, \mathrm{peakVO}_{2}\right.$, ventilation/carbon dioxide relationship slope, sodium, kidney function, hemoglobin, beta-blockers and digoxin)
\end{abstract}

\footnotetext{
* Corresponding author at: Centro Cardiologico Monzino, IRCCS, Via Parea 4, 20138 Milan, Italy. Tel.: + 390258002586 ; fax: + 390258002283.

E-mail address: piergiuseppe.agostoni@unimi.it (P. Agostoni).
} 
AF was no longer associated with adverse outcomes. Matching procedure resulted in 338 couples. CV death and HTX occurred in 63 (18.6\%) AF vs. 74 (21.9\%) SR ( $p=0.293)$ and all-cause death in $71(21 \%)$ AF vs. 80 (23.6\%) SR $(\mathrm{p}=0.406)$, with no survival differences between groups.

Conclusion: In systolic HF AF is a marker of disease severity but not an independent prognostic indicator. (c) 2015 European Federation of Internal Medicine. Published by Elsevier B.V. All rights reserved.

\section{Introduction}

Atrial fibrillation (AF) is common in patients affected by chronic heart failure (HF) [1,2]. Data from the EuroHeart Failure Survey reported that about $20 \%$ of patients with $\mathrm{HF}$ exhibit $\mathrm{AF}$ and that its prevalence reaches $40 \%$ in patients with advanced disease [3,4]. HF patients with AF show a more adverse prognosis compared to patients with HF in sinus rhythm (SR) [5-7]. Occurrence of AF in patients with HF worsens symptoms and complicates therapeutic management, due to several detrimental effects including heart rate increase, reduced left ventricular (LV) loading, irregular periods of ventricular filling and decreased cardiac output [8]. Yet, it remains unclear whether the association of $\mathrm{AF}$ and prognosis in HF is due to the consequences of the arrhythmia itself or to the fact that AF is usually associated with a more severe clinical status. Conflicting results on the prognostic role of AF in HF patients have been recently reported [9-13].

In this study we investigated the prognostic role of $\mathrm{AF}$ in a large, multicenter Italian population of patients with chronic HF and reduced LV ejection fraction (EF) that underwent cardiopulmonary exercise testing (CPET). All patients are part of the Metabolic Exercise test data combined with Cardiac and Kidney Indexes (MECKI) score database [14-16]. To this aim, we compared the outcome of HF patients with and without $\mathrm{AF}$ in the overall study population, as previously done in literature. Moreover, using a novel approach, we performed a matching analysis for established HF prognostic parameters to assess, independently of confounding factors, the prognostic role of AF in patients with systolic HF.

\section{Methods}

\subsection{Population and study procedures}

We performed a cohort study on 3447 patients with a history of HF with reduced LVEF, enrolled and prospectively followed in 17 Italian HF centers. Most of the patients (2716) were derived from a previous study that validated a new prognostic HF risk model, the MECKI score [14]. Remaining patients derived from a recruitment extension of the MECKI score database.

As previously reported [14], patients needed to fulfill the following criteria to be included in the study: previously recorded or present HF symptoms (NYHA classes I-III, stage C of ACC/AHA classification) and former certification of LV systolic impairment (LVEF $<40 \%$ ), stable HF therapy for at least three months, ability to perform a CPET at maximal effort and no major cardiovascular (CV) intervention or invasive treatment scheduled. Exclusion criteria were moderate to severe mitral and aortic stenosis, active pericardial disease, history of pulmonary embolism and severe obstructive pulmonary disease. At enrollment, inclusion and exclusion criteria were evaluated and clinical history was recorded. Then, physical examination, laboratory, ECG and transthoracic echocardiography were performed [14]. CPETs were performed, depending on the enrolling center's equipment, using a ramp protocol on electronically braked cycle-ergometer or a Bruce modified protocol on treadmill technology. Peak oxygen uptake $\left(\mathrm{VO}_{2}\right)$ data measured at treadmill exercise were reduced by $10 \%$ to allow comparison with cycle-ergometer [17]. CPET protocol was set to reach peak exercise between 8 and 12 minutes but tests were stopped as patients reported maximal effort [18]. Exercise parameters were calculated as previously described [14]. Patients were classified as having AF or not according to the rhythm present on the baseline ECG [19-21]. Patients with sinus rhythm at baseline, but with a history of AF were considered to have paroxysmal AF and so included in the no-AF group. The study complies with the Declaration of Helsinki, the local ethics committee has approved the research protocol and informed consent has been obtained from patients.

\subsection{Follow-up and study endpoints}

Patients' follow-up and procedures of data management were performed as previously described [14]. In brief follow-up was carried out according to the local HF program and ended with the last clinical evaluation or with patients' death, heart transplantation or LV assist device implantation. If a patient died outside the hospital where he/she was followed up, medical records of the event and reported cause of death were considered. The primary study endpoint was the composite of CV death and heart transplantation. In addition, a survival analysis considering the endpoint of all-cause death was also performed.

\subsection{Data analysis}

Data analysis was performed in two steps. For the first we considered the entire population and divided patients into two groups according to the presence or not of AF. Demographic, laboratory, echocardiographic, CPET and follow-up data were compared between groups, and Cox univariable and multivariable regression analysis and Kaplan-Meier analysis were performed for the previously described study endpoints. In the second step, as a confirmation of the first analysis, we performed a 1:1 statistical matching between SR and AF patients. Groups were matched for the following variables: age \pm 5 years, gender, $\mathrm{LVEF} \pm 5$, peak $\mathrm{VO}_{2} \pm 3(\mathrm{ml} / \mathrm{min} / \mathrm{kg})$ and recruiting center. Three hundred thirty-eight couples were found in whom demographic, laboratory, echocardiographic, CPET and follow-up data were compared. Then, Kaplan Meir survival analysis was repeated as previously described.

\subsection{Statistical analysis}

Numerical variables were reported as mean \pm SD or as median and interquartile range when non-normally distributed. Unpaired $t$-test or non-parametric Wilcoxon tests were used when appropriate for between-group comparison. Categorical variables, expressed as percentage or frequency, were compared by chi-square test. Potential predictors of the study outcomes were identified by univariable Cox regression analysis. A multivariable Cox proportional hazard model was used for assessing the independent prognostic value of AF adjusted for the variables significant at the univariable analysis. AF patients were matched with SR patients through a 1:1 statistical matching. This method consists of searching in the "control" group patients with conditions similar to the ones of the "test" group, in order to reduce the presence of confounding factors that may affect the comparison between groups. Hazard ratios and 95\% confidence intervals were calculated. Survival analysis was evaluated through Kaplan Meier analysis and compared by Log-Rank test. A p value $<0.05$ was used to define statistical significance. All analyses were performed using SAS 9.2 statistical package (SAS Institute Inc., Cary, NC, USA) and IBM SPSS Statistics version 20. 


\section{Results}

We studied $3447 \mathrm{HF}$ patients (85\% males) with mean age of $61.5 \pm$ 11.8 years and median LVEF of 34.9\% (interquartile range 25-37). Patients were followed for a median period of 3.15 years (interquartile range 1.51-5.24) and 513 CV deaths and 72 heart transplantations were recorded.

\subsection{Comparison of AF vs. SR in the overall population}

Of the 3447 patients, 16\% (565) had AF and 84\% (2882) were in SR. Patients' demographic, laboratory, echocardiographic, CPET and follow-up data are reported in Table 1 . As in previous studies [15,22, 23], AF patients were older, showed lower peakVO ${ }_{2}$, more severe NYHA class and were more often treated with oral anticoagulant therapy, digoxin and amiodarone. In particular, $77 \%$ of $\mathrm{AF}$ patients were on treatment with oral anticoagulants, while the remaining $23 \%$ were excluded from this kind of therapy due to the presence of contraindications to anticoagulants or to patients' inability to manage them based on referring cardiologist judgment. In the AF group, idiopathic dilated cardiomyopathy was more often the etiology of HF than in SR patients, and a worse renal function was observed. CV death and cardiac transplantation occurred in 114 (20\%) AF patients compared to $471(16 \%)$ SR patients $(\mathrm{p}=0.026)$. In patients with AF, $130(23 \%)$ deaths from

Table 1

Heart failure patients' characteristics considering overall study population divided in the two groups of AF and SR.

\begin{tabular}{|c|c|c|c|}
\hline & $\begin{array}{l}\mathrm{AF}^{\mathrm{a}} \\
(\mathrm{n}=565)\end{array}$ & $\begin{array}{l}\mathrm{SR}^{\mathrm{b}} \\
(\mathrm{n}=2882)\end{array}$ & p Value \\
\hline Age (yrs) & $65.7 \pm 10.7$ & $60.7 \pm 11.8$ & $<0.001$ \\
\hline Gender (males,\%) & $486(86)$ & $2431(84)$ & ns \\
\hline Body mass index $\left(\mathrm{kg} / \mathrm{m}^{2}\right)$ & $26.8 \pm 4.3$ & $26.7 \pm 4.3$ & ns \\
\hline NYHA ${ }^{\mathrm{c}}$ class & $2.4 \pm 0.6$ & $2.2 \pm 0.7$ & $<0.001$ \\
\hline $\begin{array}{l}\text { Ischemic dilated cardiomyopathy } \\
(\mathrm{n}, \%)\end{array}$ & $226(40)$ & $1505(52)$ & $<0.001$ \\
\hline $\begin{array}{l}\text { Idiopathic dilated cardiomyopathy } \\
(\mathrm{n}, \%)\end{array}$ & $262(47)$ & $1150(40)$ & $<0.001$ \\
\hline Other etiology $(\mathrm{n}, \%)$ & $74(13 \%)$ & $225(8)$ & $<0.001$ \\
\hline $\operatorname{ICD}^{\mathrm{d}}(\mathrm{n}, \%)$ & $118(21)$ & $734(26)$ & 0.012 \\
\hline $\mathrm{CRT}^{\mathrm{e}}(\mathrm{n}, \%)$ & $55(10)$ & $267(10)$ & ns \\
\hline $\operatorname{LVEF}^{f}(\%)$ & $31.9(25-38.1)$ & $30(25-37)$ & 0.022 \\
\hline Creatinine $(\mathrm{mg} / \mathrm{dl})$ & $1.2(0.97-1.46)$ & $1.1(0.91-1.32)$ & $<0.001$ \\
\hline $\mathrm{MDRD}^{\mathrm{g}}$ & $65.5 \pm 22$ & $71.1 \pm 23.1$ & $<0.001$ \\
\hline Sodium (mEq/l) & $139.3 \pm 3.5$ & $139.4 \pm 3.3$ & ns \\
\hline Potassium $(\mathrm{mEq} / \mathrm{l})$ & $4.3 \pm 0.4$ & $4.2 \pm 0.5$ & $<0.01$ \\
\hline Hemoglobin $(\mathrm{g} / \mathrm{dl})$ & $13.5 \pm 1.7$ & $13.5 \pm 1.6$ & ns \\
\hline Peak $\mathrm{VO}_{2}{ }^{\mathrm{h}}(\mathrm{ml} / \mathrm{kg} / \mathrm{min})$ & $13.0(10.4-16)$ & $14.2(11.6-17.3)$ & $<0.001$ \\
\hline $\mathrm{ACE}^{\mathrm{i}}$-inhibitor $(\mathrm{n}, \%)$ & $407(72)$ & $2271(79)$ & $<0.001$ \\
\hline $\begin{array}{l}\text { Angiotensin II receptors blocker } \\
\quad(\mathrm{n}, \%)\end{array}$ & $94(17)$ & $439(15.3)$ & ns \\
\hline Beta-blocker (n, \%) & $415(73)$ & $2412(84)$ & $<0.001$ \\
\hline Diuretics (n, \%) & $487(86)$ & $2312(80)$ & 0.001 \\
\hline Anti-aldosteronic drugs ( $\mathrm{n}, \%)$ & $320(57)$ & $1523(53)$ & ns \\
\hline Digoxin (n, \%) & $252(45)$ & $588(20)$ & $<0.001$ \\
\hline Amiodarone $(\mathrm{n}, \%)$ & $178(32)$ & $720(25)$ & 0.001 \\
\hline Aspirin (n, \%) & $136(24)$ & $1687(59)$ & $<0.001$ \\
\hline Anticoagulant oral therapy (n, \%) & $435(77)$ & $648(23)$ & $<0.001$ \\
\hline Follow-up (yrs) & $3.01(1.53-5.31)$ & $3.20(1.50-5.24)$ & ns \\
\hline All-cause death (\%) & $130(23)$ & $554(19.2)$ & 0.039 \\
\hline $\mathrm{CV}^{\mathrm{j}}$ death + cardiac transplant (\%) & $114(20)$ & $471(16)$ & 0.026 \\
\hline
\end{tabular}

Data are expressed as mean value $\pm \mathrm{SD}$, median values and interquartile range or number (\%) of patients.

a $\mathrm{AF}=$ atrial fibrillation

b $\mathrm{SR}=$ sinus rhythm.

c NYHA $=$ New York Heart Association

d ICD = implantable cardioverter defibrillator.

e $\mathrm{CRT}=$ cardiac resynchronization therapy.

${ }^{\mathrm{f}} \mathrm{LVEF}=$ left ventricular ejection fraction.

g $\mathrm{MDRD}=$ modification of diet in renal disease.

h $\mathrm{VO}_{2}=$ oxygen uptake.

i $\mathrm{ACE}=$ Angiotensin Converting Enzyme.

${ }^{\mathrm{j}} \mathrm{CV}=$ cardiovascular.
A)

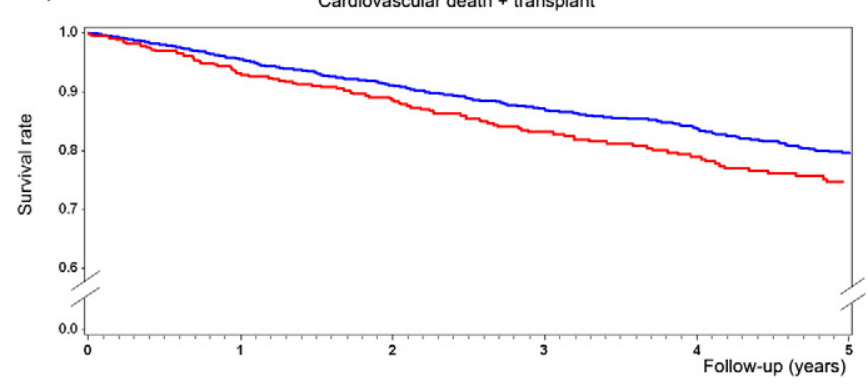

B)

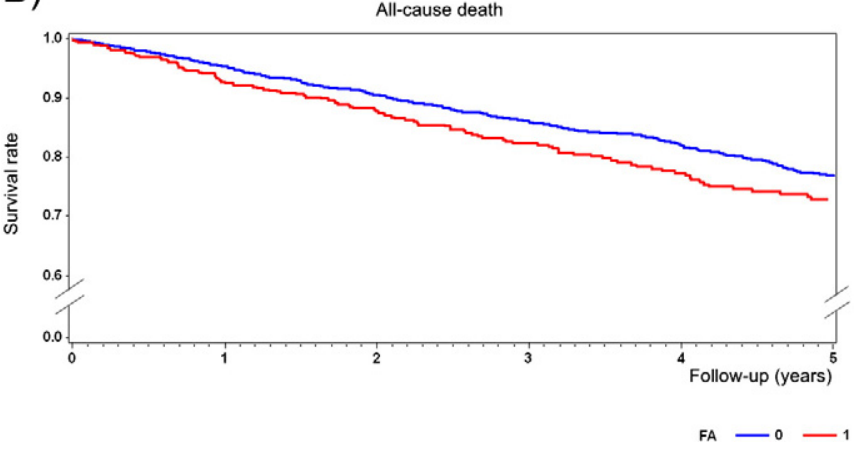

Fig. 1. Kaplan-Meier analysis in the overall study population divided into two groups of AF $(\mathrm{n}=565)$ and SR $(\mathrm{n}=2882)$ patients. A. Kaplan-Meier analysis for the composite outcome of CV death + cardiac transplantation. B. Kaplan-Meier analysis for the endpoint of all-cause death.

any cause were recorded compared to 554 (19.2\%) in the SR group $(\mathrm{p}=0.039)$. Kaplan-Meier analysis showed a significantly worse prognosis of the AF group for the composite outcome of CV death + cardiac transplantation (Log-Rank $\mathrm{p}=0.045$; Fig. $1 \mathrm{~A}$ ), while differences did not reach statistical significance for any-cause death ( Log-Rank $\mathrm{p}=0.065$; Fig. 1B).

\subsection{Prognostic value of AF in the overall study population: multivariable model}

In all patients, at univariable Cox analysis, the presence of AF was significantly related to prognosis, both for the composite outcome of CV death and heart transplantation (HR 1.235, 95\% CI 1.017-1.5; ProbChiSq $=0.032$ ) and all-cause death (HR 1.284, 95\% CI 1.0471.574; ProbChiSq = 0.016). In addition, several of the analyzed variables resulted significantly related to prognosis. When variables were analyzed in a multivariable Cox model, AF was no longer associated with adverse outcome (Table 2, Table 3).

Table 2

Multivariable Cox regression analysis in overall study population for the primary composite outcome of cardiovascular death and heart transplantation.

\begin{tabular}{llllr}
\hline & HR & Lower CI & Upper CI & ProbChiSq \\
\hline Gender & 1.640 & 1.205 & 2.232 & 0.001 \\
$\mathrm{LVEF}^{\mathrm{a}}$ & 0.966 & 0.952 & 0.980 & $<0.001$ \\
$\mathrm{VO}_{2}{ }^{\mathrm{b}}$ peak $(\mathrm{ml} / \mathrm{Kg} / \mathrm{min})$ & 0.929 & 0.899 & 0.960 & $<0.001$ \\
${\mathrm{VE} / \mathrm{VCO}_{2}{ }^{\mathrm{c}} \text { slope }}_{\text {Sodium }}$ & 1.030 & 1.017 & 1.043 & $<0.001$ \\
MDRD $^{\mathrm{d}}$ & 0.955 & 0.927 & 0.983 & 0.001 \\
Hemoglobin & 0.989 & 0.984 & 0.994 & $<0.001$ \\
Beta-blockers & 0.876 & 0.820 & 0.936 & $<0.001$ \\
Digoxin & 0.750 & 0.596 & 0.943 & 0.014 \\
\hline
\end{tabular}

${ }^{\text {a }} \mathrm{LVEF}=$ left ventricular ejection fraction.

b $\mathrm{VO}_{2}=$ oxygen uptake.

c $\mathrm{VE} / \mathrm{VCO}_{2}=$ minute ventilation/carbon dioxide production slope.

${ }^{\mathrm{d}} \mathrm{MDRD}=$ modification of diet in renal disease. 
Table 3

Multivariable Cox regression analysis in overall study population for the study endpoint of all-cause death.

\begin{tabular}{llllr}
\hline & HR & Lower CI & Upper CI & ProbChiSq \\
\hline Gender & 1.706 & 1.278 & 2.277 & $<0.001$ \\
LVEF $^{\mathrm{a}}$ & 0.973 & 0.960 & 0.986 & $<0.001$ \\
$\mathrm{VO}_{2}{ }^{\mathrm{b}}$ peak $(\mathrm{ml} / \mathrm{Kg} / \mathrm{min})$ & 0.936 & 0.908 & 0.964 & $<0.001$ \\
${\mathrm{VE} / \mathrm{VCO}_{2}{ }^{\mathrm{c}} \text { slope }}_{\text {Sodium }}$ & 1.030 & 1.018 & 1.042 & $<0.001$ \\
$\mathrm{MDRD}^{\mathrm{d}}$ & 0.955 & 0.930 & 0.982 & 0.001 \\
Hemoglobin & 0.991 & 0.986 & 0.996 & $<0.001$ \\
Beta-blockers & 0.870 & 0.817 & 0.925 & $<0.001$ \\
Digoxin & 0.791 & 0.638 & 0.981 & 0.032 \\
\hline
\end{tabular}

Abbreviations:

a $\mathrm{LVEF}=$ left ventricular ejection fraction.

b $\mathrm{VO}_{2}=$ oxygen uptake.

c $\mathrm{VE} / \mathrm{VCO}_{2}=$ minute ventilation/carbon dioxide production slope.

${ }^{d}$ MDRD $=$ modification of diet in renal disease.

\subsection{Comparison of AF vs SR in matched groups}

Using matching criteria, 338 couples of AF and SR patients were identified. Patients' demographic, laboratory, echocardiographic, CPET and follow-up data are reported in Table 4. As expected, AF patients were more treated with oral anticoagulant therapy and digoxin, and, as observed in the overall population, they showed worse functional NYHA class and idiopathic dilated cardiomyopathy as more frequent etiology of HF. CV death and cardiac transplantation occurred in 63 (18.6\%) AF patients compared to 74 (21.9\%) SR patients $(\mathrm{p}=0.293$ ).

Table 4

$\mathrm{AF}$ and SR patients' characteristics after matching for age gender, $\mathrm{LVEF}$, peak $\mathrm{VO}_{2}$ and enrolling center (338 couples).

\begin{tabular}{|c|c|c|c|}
\hline & $\begin{array}{l}\mathrm{AF}^{\mathrm{a}} \\
(\mathrm{n}=338)\end{array}$ & $\begin{array}{l}\mathrm{SR}^{\mathrm{b}} \\
(\mathrm{n}=338)\end{array}$ & $\mathrm{p}$ \\
\hline Body mass index $\left(\mathrm{kg} / \mathrm{m}^{2}\right)$ & $26.6 \pm 4$ & $26.7 \pm 4$ & $\mathrm{~ns}$ \\
\hline $\mathrm{NYHA}^{\mathrm{c}}$ class & $2.3 \pm 0.6$ & $2.1 \pm 0.6$ & $<0.001$ \\
\hline $\begin{array}{l}\text { Ischemic dilated cardiomyopathy } \\
\quad(\mathrm{n}, \%)\end{array}$ & $141(42)$ & $202(60)$ & $<0.001$ \\
\hline $\begin{array}{l}\text { Idiopathic dilated cardiomyopathy } \\
(\mathrm{n}, \%)\end{array}$ & $158(47)$ & $108(32)$ & $<0.001$ \\
\hline Other etiology (n, \%) & $37(11 \%)$ & $28(8)$ & $<0.001$ \\
\hline $\mathrm{ICD}^{\mathrm{d}}(\mathrm{n}, \%)$ & $78(23)$ & $93(28)$ & ns \\
\hline $\mathrm{CRT}^{\mathrm{e}}(\mathrm{n}, \%)$ & $37(12)$ & $37(12)$ & ns \\
\hline Creatinine $(\mathrm{mg} / \mathrm{dl})$ & $1.2(1-1.51)$ & $1.2(0.98-1.44)$ & ns \\
\hline $\operatorname{MDRD}^{\mathrm{f}}$ & $64.7 \pm 22.4$ & $66.3 \pm 23.7$ & ns \\
\hline Sodium (mEq/l) & $139.2 \pm 3.5$ & $139.3 \pm 3.4$ & ns \\
\hline Potassium (mEq/l) & $4.3 \pm 0.5$ & $4.4 \pm 0.5$ & 0.005 \\
\hline Hemoglobin (g/dl) & $13.6 \pm 1.6$ & $13.4 \pm 1.7$ & ns \\
\hline $\mathrm{ACE}^{\mathrm{g}}$-inhibitor $(\mathrm{n}, \%)$ & $248(73)$ & $266(79)$ & ns \\
\hline $\begin{array}{l}\text { Angiotensin II receptors blocker } \\
(\mathrm{n}, \%)\end{array}$ & $51(15)$ & $45(13)$ & ns \\
\hline Beta-blocker (n, \%) & $250(74)$ & $272(80)$ & 0.044 \\
\hline Diuretics (n, \%) & $288(85)$ & $291(86)$ & ns \\
\hline Anti-aldosteronic drugs ( $\mathrm{n}, \%)$ & $191(57)$ & $182(54)$ & ns \\
\hline Digoxin $(n, \%)$ & $133(39)$ & $64(19)$ & $<0.001$ \\
\hline Amiodarone (n, \%) & $107(32)$ & $103(31)$ & ns \\
\hline Aspirin (n, \%) & $92(27)$ & $208(62)$ & $<0.001$ \\
\hline Anticoagulant oral therapy (n, \%) & $257(76)$ & $70(21)$ & $<0.001$ \\
\hline Follow-up (yrs) & $2.84(1.6-5.31)$ & $3.04(1.27-5.07)$ & ns \\
\hline All-cause death $(\%)$ & $71(21)$ & $80(23.6)$ & ns \\
\hline $\mathrm{CV}^{\mathrm{h}}$ death + cardiac transplant $(\%)$ & $63(18.6)$ & $74(21.9)$ & ns \\
\hline
\end{tabular}

Data are expressed as mean value \pm SD, median values and interquartile range or number

(\%) of patients.

Abbreviations:

a $\mathrm{AF}=$ atrial fibrillation

b $\mathrm{SR}=$ sinus rhythm.

c $\mathrm{NYHA}=$ New York Heart Association.

d ICD = implantable cardioverter defibrillator.

e $\mathrm{CRT}=$ cardiac resynchronization therapy.

f MDRD = modification of diet in renal disease.

g $\mathrm{ACE}=$ Angiotensin Converting Enzyme.

h $\mathrm{CV}=$ cardiovascular.
A)

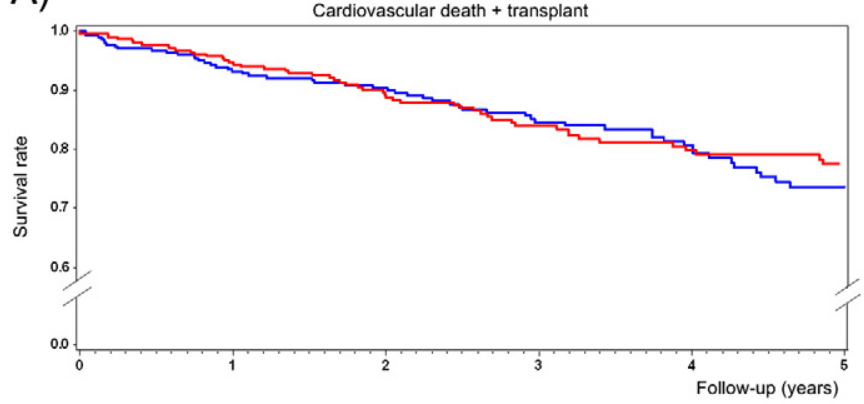

B)

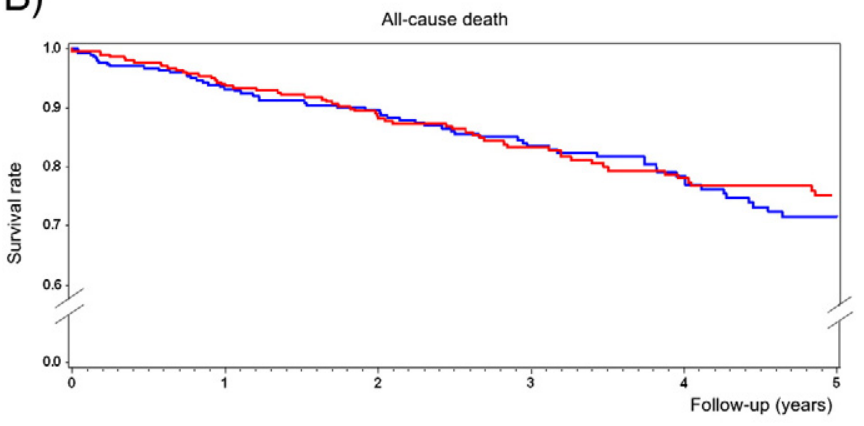

FA $-0-1$

Fig. 2. Kaplan-Meier analysis in the matched groups (338 couples) of AF and SR patients. A. Kaplan-Meier analysis for the composite outcome of CV death + cardiac transplantation. B. Kaplan-Meier analysis for the endpoint of all-cause death.

In patients with $\mathrm{AF}, 71$ (21\%) deaths from any cause were recorded compared to $80(23.6 \%)$ events in SR patients $(\mathrm{p}=0.406)$. KaplanMeier analysis showed no differences between AF and SR patients both for CV death + cardiac transplantation (Log-rank $\mathrm{p}=0.382$; Fig. 2A) and all-cause death (Log-Rank $\mathrm{p}=0.556$; Fig. 2B).

\section{Discussion}

The present study confirms that persistent or permanent AF is frequent in chronic HF with reduced LVEF and supports the hypothesis that $\mathrm{AF}$ is not independently associated with adverse HF prognosis, either in the whole cohort, or in a subgroup of patients with AF or SR matched for clinical characteristics and follow-up.

Novelties of the present study are represented by the examined HF population and by the statistical approach used to investigate the prognostic role of AF in HF. Concerning the study population, we analyzed relatively young patients with, in particular, 77 moderate systolic impairment, mean II NYHA class and ability to perform a CPET at maximal effort. Still contrasting data are available about the prognostic role of $\mathrm{AF}$ in HF patients with modest functional impairment and moderate to severe LV dysfunction. However, no data about exercise capacity were reported in previous studies and exercise related variables were not included in regression models, despite their well-known prognostic value in HF patients. About the statistical approach, a standard multivariable model and a case-matching process, which aimed to match patients with AF and SR for potential confounding characteristics, were performed to assess the prognostic role of AF in HF. Despite the application of these two approaches might appear as a repetition, we performed both analyses to overcome methods' limitations and to add a novel methodology compared to previous reports in which only a multivariable model wad applied [9,13,19-21,25].

\subsection{Previous studies}

To date, there is no consensus on the prognostic role of AF in patients affected by chronic, systolic HF. Two meta-analyses [6,7], including 
53,969 and 32,946 patients, reported that the presence of AF in chronic $\mathrm{HF}$ is associated with a $30-40 \%$ increased risk of mortality. However, in both studies, significant demographic and clinical differences were observed between patients with AF or SR, although the analyses were not adjusted for potential confounders. An independent prognostic role of AF has been reported in a retrospective analysis of the SOLVD study [19], which included 6517 patients with reduced LVEF. Similarly, in a CHARM study subanalysis [20], the presence of AF was associated, at multivariate analysis, with an increased risk of all-cause mortality and CV death/hospitalization. On the contrary, data from 3029 chronic HF patients enrolled in the COMET study [21] revealed that, after adjusting for other predictors of prognosis, AF was no longer an independent predictor of mortality. However, new onset AF was indpendently associated with a worse prognosis. Data from V-HeFT I and II trials [24] demonstrated that the presence of AF in patients with mild to moderate systolic HF does not affect morbidity and mortality rates.

More recently, Tveit et al. [25] analyzed data from 4048 patients referred to Norwegian HF outpatient clinics, 1391 of which were in AF and 2657 in SR, followed-up for 28 months, and found that AF was associated with an increased risk of death at univariate analysis $(\mathrm{HR}=1.181$; $\mathrm{p}=0.008$ ), but that this effect disappeared when adjusted for confounding variables, such as age, NYHA class and ischemic HF etiology (HR = 1.037; $\mathrm{p}=0.619$ ). Similar results were reported from the Japanese Cardiac Registry on HF in Cardiology (JCARE-CARD) [13], where AF was non independently associated with all-cause death as well as with hospitalization for HF. Raunso et al. [11] followed up to 7 years 2881 patients hospitalized for HF and found that AF was associated with an increased risk of death only in presence of an underlying ischemic etiology, whereas, Linssen et al. [12] reported that AF was an independent predictor of the composite outcome of death or HF hospitalization only in patients affected by HF with preserved LVEF (HR = 1.49; $\mathrm{p}=0.03$ ) but not in those with reduced systolic function ( $H R=1.05 ; p=0.72$ ). Finally it should be noticed that the population analyzed in the present study is characterized by HF patients able to perform a symptom limited CPET. Accordingly our population differs from all the previous ones.

In the entire study population of the present study, univariable analysis showed a more frequent occurrence of the composite outcome of CV death and heart transplantation $(20 \%$ vs. $16 \%$; $p=0.026)$ and higher rates of all-cause mortality ( $23 \%$ vs. $19.2 \%$; $p=0.039$ ) in patients with AF. Nevertheless, HF patients with AF, compared to SR patients, significantly differed for several characteristics that are associated with adverse prognosis, including age, NYHA class, peak $\mathrm{VO}_{2}$ and renal function, making it difficult to distinguish an independent prognostic influence of AF on adverse outcomes. To overcome these differences, we analyzed the independent impact of AF using two different statistical models: 1) a multivariate, Cox regression model assessing the independent role of AF in the whole study cohort; and 2) a matching analysis considering demographic data (age and gender), HF severity parameters ( $\mathrm{LVEF}$, peakVO $\mathrm{H}_{2}$ ) in a subgroup of patients with either AF or SR. Notably matching analysis included the center where the patients were recruited. This is particularly important because it allowed us to overcome possible differences in treatment and follow-up strategies as well as in referred population, which are likely to occur in multicenter studies, and might have affected patients' outcome. To our knowledge, this is the first analysis matching AF and SR patients with chronic HF that enables more accurately to investigate the independent role of AF in chronic HF patients. Both models showed that AF was not an independent predictor of adverse outcome in our patients with systolic HF.

\subsection{Study limitations}

The results of the present study are in line with the MECKI score [14], where only 6 out of the almost 200 variables analyzed resulted related to prognosis - specifically peak $\mathrm{VO}_{2}$, LVEF, hemoglobin, plasma $\mathrm{Na}^{+}$, kidney function as assessed by MDRD, and ventilation efficiency during exercise as assessed by the minute ventilation vs. carbon dioxide production slope. In further studies of the MECKI score research group, the only variable that adds a prognostic power to the MECKI score was the non-identification of anaerobic threshold [16]. In this regard, a relevant methodological study limitation must be recognized. Indeed, our population, albeit much larger, included HF patients used for the MECKI score calculation. This is a strength, but at the same time a potential weakness of the study, which selected patients with specific criteria [14], including the ability to perform a cardiopulmonary stress test. In addition, MECKI score study was not designed to prospectively assess the importance of AF in HF, so the importance of AF may be underestimated in this analysis. Although we did not identify a specific prognostic role of $\mathrm{AF}$ in $\mathrm{HF}$, our observation does not add any information about the management of $\mathrm{AF}$ in HF, including the opportunity/ need for cardioversion or to the debate between rhythm control and rate control. Interestingly, it is unknown at present if SR restoration is associated with changes of parameters such as LVEF or peak $\mathrm{VO}_{2}$ that offset the direct role of AF in HF prognosis. About the effects of antiarrhythmic drugs on study outcomes, we also repeated the multivariable analysis including drugs and no effects on the study outcomes were observed for HF treatments or for reported antiarrhythmic drugs (data not shown). Notably in the first MECKI score study [14] amiodarone, the most used antiarrhythmic drug in HF patients, was significantly related to prognosis only at univariate analysis. The likely explanation is that this drug is useful but given usually to patients with most severe HF.

Other study limitations must be considered. First of all, no data are available about the prognostic role of new onset AF in HF patients and no analysis for AF subtypes was performed. In addition, our follow-up was particularly long - an average of 3.15 years. Again, this is a strength of the present study, but also a weakness. Indeed, during follow-up, clinical, laboratory and therapeutic changes may have occurred; also AF may have shown up in some cases and disappeared in others - albeit unlikely for persistent and permanent AF. Also, we recognize that we have considered only 5 of the several confounding factors in the matching analyses. Indeed, although ours was a large population with a prolonged follow-up, adding other matching categories would have reduced the couples available and therefore reduced the significance of our study. However, because also considering 5 matching variables the prognostic significance of $\mathrm{AF}$ in $\mathrm{HF}$ was lost, we believe that this is a negligible limitation. Among analyzed variables, we did not include QRS duration because of lack of this data from some of the recruiting centers. However, in an ongoing update of our database we are also trying to collect information on this parameter in order to assess its prognostic value in AF and SR patients. Finally, in the present study as well as in others [19], AF was more frequently observed in patients with idiopathic cardiomyopathy. We have not a definite explanation for this finding. Moreover, due to the small number of patients with idiopathic cardiomyopathy we couldn't analyze these patients separately.

\section{Conclusion}

This large, multicenter study showed that AF is a marker of advanced disease, but it is not independently associated to adverse prognosis in chronic HF patients with reduced LVEF.

\section{Learning points}

- Atrial fibrillation is common in patients affected by chronic heart failure, however it remains unclear whether the association of atrial fibrillation and prognosis in heart failure is due to the consequences of the arrhythmia itself or to the fact that atrial fibrillation is usually associated with a more severe clinical status.

- The results of the current study confirm that persistent or permanent atrial fibrillation is frequent in chronic heart failure with reduced systolic function and demonstrates that it is not independently associated with adverse heart failure prognosis, either in the whole analyzed population, or in a subgroup of patients with atrial fibrillation or 
sinus rhythm matched for clinical characteristics and follow-up, whereas should be considered as a marker of advanced disease.

\section{Conflict of interest}

The authors state that they have no conflicts of interest.

\section{Source of funding}

None.

\section{Appendix A}

Other members of the MECKI score group are:

- Centro Cardiologico Monzino, IRCCS, Milano: Fabrizio Veglia, Anna Apostolo, Pietro Palermo, Stefania Farina, Mauro Contini, Valentina Mantegazza, Erika Bertella;

- Department of Clinical and Molecular Medicine, Azienda Ospedaliera Sant'Andrea, "Sapienza" Università degli studi di Roma, Italy: Damiano Magrì, Matteo Casenghi;

- Cardiac Rehabilitation Unit, Fondazione Salvatore Maugeri, IRCCS, Scientific Institute of Milan, Milan, Italy: Maurizio Bussotti, Giovanni Marchese;

- Fondazione Salvatore Maugeri, IRCCS, Istituto Scientifico di Veruno: Pantaleo Giannuzzi, Andrea Giordano, Alessandro Mezzani;

- Cardiology Division, Santo Spirito Hospital, Roma, Italy: Alessandro Ferraironi, Roberto Ricci;

- Cardiologia Riabilitativa, Ospedali Riuniti, Ancona: Francesca Pietrucci;

- Istituto Auxologico Italiano: Gabriella Malfatto;

- Istituto Mediterraneo per i Trapianti e Terapie ad Alta Specializzazione, ISMETT, Palermo, Italy: Chiara Minà;

- Cardiologia SUN, Ospedale Monaldi, Napoli: Teo Roselli, Andrea Buono, Raffaele Calabrò;

- CNR-Milano: Renata De Maria;

- "S. Maugeri" Foundation, IRCCS, Cassano Murge: Andrea Passantino, Daniela Santoro, Saba Campanale, Domenica Caputo;

- "S. Maugeri" Foundation, Tradate: Raffaella Vaninetti, Donatella Bertipaglia;

- Ospedali Riuniti and University of Trieste: Marco Confalonieri, Emanuela Berton, Chiara Torregiani;

- Dipartimento Cardiologico “A. De Gasperis”, Ospedale Cà GrandaA.O. Niguarda, Milano, Italy: Fabrizio Oliva;

- Fondazione Gabriele Monasterio, CNR-Toscana, Pisa: Luigi E Pastormerlo;

- Cardiology, University of Civil Hospital, Brescia: Livio Dei Cas, Valentina Carubelli;

- Section of Cardiology, Department of Medicine, University of Verona: Elisa Battaia, Corrado Vassanelli;

- Division of Cardiology, Salvatore Maugeri Foundation, IRCCS, Institute of Milan: Giovanni Marchese;

- Southampton Statistical Sciences Research Institute \& School of Mathematics, University of Southampton, Southampton, United Kingdom; Department of Public Institutions, Economy and Society, Università "Roma Tre", Roma, Italy: Antonello Maruotti;

- Department of Cardiology, University of Foggia: Antonio Totaro, Armando Ferraretti, Tommaso Passero.

\section{References}

[1] Maisel WH, Stevenson LW. Atrial fibrillation in heart failure: epidemiology, pathophysiology, and rationale for therapy. Am J Cardiol 2003;91:2D-8D.

[2] Nieuwlaat R, Eurlings LW, Cleland JG, Cobbe SM, Vardas PE, Capucci A, et al. Atrial fibrillation and heart failure in cardiology practice: reciprocal impact and combined management from the perspective of atrial fibrillation: results of the Euro Heart Survey on atrial fibrillation. J Am Coll Cardiol 2009;53:1690-8.
[3] Nieuwlaat R, Capucci A, Camm AJ, Olsson SB, Andresen D, Davies DW, et al. European Heart Survey investigators. Atrial fibrillation management: a prospective survey in ESC member countries: the Euro Heart Survey on atrial fibrillation. Eur Heart J $2005 ; 26: 2422-34$

[4] Cleland JG, Swedberg K, Follath F, Komajda M, Cohen-Solal A, Aguilar JC, et al. Study Group on diagnosis of the working group on heart failure of the European Society of Cardiology. The EuroHeart Failure survey programme-a survey on the quality of care among patients with heart failure in Europe. Part 1: patient characteristics and diagnosis. Eur Heart J 2003;24:442-63.

[5] Wang TJ, Larson MG, Levy D, Vasan RS, Leip EP, Wolf PA, et al. Temporal relations of atrial fibrillation and congestive heart failure and their joint influence on mortality: the Framingham Heart Study. Circulation 2003;107:2920-5.

[6] Mamas MA, Caldwell JC, Chacko S, Garratt CJ, Fath-Ordoubadi F, Neyses L. A metaanalysis of the prognostic significance of atrial fibrillation in chronic heart failure. Eur J Heart Fail 2009;11:676-83.

[7] Wasywich CA, Pope AJ, Somaratne J, Poppe KK, Whalley GA, Doughty RN. Atrial fibrillation and the risk of death in patients with heart failure: a literature-based meta-analysis. Intern Med J 2010;40:347-56.

[8] McMurray JJ, Adamopoulos S, Anker SD, Auricchio A, Böhm M, Dickstein K, et al. ESC Committee for Practice Guidelines. ESC Guidelines for the diagnosis and treatment of acute and chronic heart failure 2012: the Task Force for the Diagnosis and Treatment of Acute and Chronic Heart Failure 2012 of the European Society of Cardiology. Developed in collaboration with the Heart Failure Association (HFA) of the ESC. Eur Heart J 2012;33:1787-847

[9] Chamberlain AM, Redfield MM, Alonso A, Weston SA, Roger VL. Atrial fibrillation and mortality in heart failure: a community study. Circ Heart Fail 2011:4:740-6.

[10] Mountantonakis SE, Grau-Sepulveda MV, Bhatt DL, Hernandez AF, Peterson ED, Fonarow GC. Presence of atrial fibrillation is independently associated with adverse outcomes in patients hospitalized with heart failure: an analysis of get with the guidelines-heart failure. Circ Heart Fail 2012;5:191-201.

[11] Raunsø J, Pedersen OD, Dominguez H, Hansen ML, Møller JE, Kjaergaard J, et al. EchoCardiography and Heart Outcome Study investigators. Atrial fibrillation in heart failure is associated with an increased risk of death only in patients with ischaemic heart disease. Eur J Heart Fail 2010;12:692-7.

[12] Linssen GC, Rienstra M, Jaarsma T, Voors AA, van Gelder IC, Hillege HL, et al. Clinical and prognostic effects of atrial fibrillation in heart failure patients with reduced and preserved left ventricular ejection fraction. Eur J Heart Fail 2011;13:1111-20.

[13] Hamaguchi S, Yokoshiki H, Kinugawa S, Tsuchihashi-Makaya M, Yokota T, Takeshita A, et al. Japanese Cardiac Registry of Heart Failure in Cardiology investigators. Effects of atrial fibrillation on long-term outcomes in patients hospitalized for heart failure in Japan: a report from the Japanese Cardiac Registry of Heart Failure in Cardiology (JCARE-CARD). Circ J 2009;73:2084-90.

[14] Agostoni P, Corrà U, Cattadori G, Veglia F, La Gioia R, Scardovi AB, et al. MECKI score research group. Metabolic exercise test data combined with cardiac and kidney indexes, the MECKI score: a multiparametric approach to heart failure prognosis. Int J Cardiol 2013;167:2710-8.

[15] Agostoni P, Emdin M, Corrà U, Veglia F, Magrì D, Tedesco CC, et al. Permanent atrial fibrillation affects exercise capacity in chronic heart failure patients. Eur Heart J 2008;29:2367-72.

[16] Agostoni P, Corrà U, Cattadori G, Veglia F, Battaia E, La Gioia R, et al. MECKI score research group. Prognostic value of indeterminable anaerobic threshold in heart failure. Circ Heart Fail 2013;6:977-87.

[17] Wasserman K, Hansen JE, Sue DY, Stringer WW, Whipp BJ. Clinical exercise testing Principles of exercise testing and interpretation including pathophysiology and clinical applications. Lippincot Williams \& Wilkins; 2005 138-9.

[18] Agostoni P, Bianchi M, Moraschi A, Palermo P, Cattadori G, La Gioia R, et al. Workrate affects cardiopulmonary exercise test results in heart failure. Eur J Heart Fail 2005; 7:498-504

[19] Dries DL, Exner DV, Gersh BJ, Domanski MJ, Waclawiw MA, Stevenson LW. Atrial fibrillation is associated with an increased risk for mortality and heart failure progression in patients with asymptomatic and symptomatic left ventricular systolic dysfunction: a retrospective analysis of the SOLVD trials. J Am Coll Cardiol 1998; 32:695-703.

[20] Olsson LG, Swedberg K, Ducharme A, Granger CB, Michelson EL, McMurray JJ, et al CHARM investigators. Atrial fibrillation and risk of clinical events in chronic heart failure with and without left ventricular systolic dysfunction: results from the candesartan in heart failure-assessment of reduction in mortality and morbidity (CHARM) program. J Am Coll Cardiol 2006;47:1997-2004.

[21] Swedberg K, Olsson LG, Charlesworth A, Cleland J, Hanrath P, Komajda M, et al. Prognostic relevance of atrial fibrillation in patients with chronic heart failure on longterm treatment with betablockers: results from COMET. Eur Heart J 2005;26: 1303-8.

[22] Corell P, Gustafsson F, Schou M, Markenvard J, Nielsen T, Hildebrandt P. Prevalence and prognostic significance of atrial fibrillation in outpatients with heart failure due to left ventricular systolic dysfunction. Eur J Heart Fail 2007;9:258-65.

[23] Pardaens K, Van Cleemput J, Vanhaecke J, Fagard RH. Atrial fibrillation is associated with a lower exercise capacity in male chronic heart failure patients. Heart 1997;78: 564-8.

[24] Carson PE, Johnson GR, Dunkman WB, Fletcher RD, Farrell L, Cohn JN. The influence of atrial fibrillation on prognosis in mild to moderate heart failure. The V-HeFT studies. The V-HeFT VA cooperative studies group. Circulation 1993;87:VI102-10.

[25] Tveit A, Flonaes B, Aaser E, Korneliussen K, Froland G, Gullestad L, et al. No impact of atrial fibrillation on mortality risk in optimally treated heart failure patients. Clin Cardiol 2011;34:537-42. 